\title{
Prolapso genital en el Hospital Universitario de Cartagena
}

\author{
Alvaro Monterrosa Castro*; Néstor Llinás Quintero, Devight Marrugo García**
}

\begin{abstract}
RESUMEN: EI Prolapso genital es una condición frecuente en la práctica clínica, definido como el descenso de las estructuras genitales y/o de elementos relacionados con él, por debajo del nivel que normalmente ocupan. La fascia endopélvica une los órganos a las paredes pélvicas, mientras los músculos del piso pélvico, cierran la pelvis y elevando los órganos, evitan la distensión de los ligamentos aponeuróticos. 102 pacientes que fueron ingresadas al Hospital Universitario de Cartagena con prolapso genital son estudiadas. Edades: $32-80$ años. $X^{-}=$ $\mathbf{5 5 . 8} \pm \mathbf{1 2 . 3}$ años. $\mathbf{7 4 . 5} \%$ tenían prolapso Grado III. La paridad promedio fue de 7 partos y ninguna era nulípara. El tipo de prolapso más frecuente fue el cistorectocele, seguido del prolapso genital total. El 30.2\% de las cirugías ginecológicas que se realizan en este Hospital son procedimientos por corrección del prolapso genital. No se presentaron accidentes intraoperatorios y las complicaciones a los 30 días postquirúrgicos fueron del $\mathbf{1 1 . 8 \%}$. La estancia hospitalaria fue de 3-8 días. El origen de los transtornos del piso pélvico se considera multifactorial, dándose gran valor al traumatismo obstétrico y más recientemente a los estados hipoestrogénicos post-menopáusicos. Hacen falta más estudios para precisar la influencia de los estados nutricionales y la tendencia familiar en el prolapso genital.
\end{abstract}

PALABRAS CLAVES: Prolapso genital, cistocele, histerocele, rectocele, histerectomía vaginal, colpoperineorrafía, desgarro perineal, traumatismo perineal.

SUMMARY: Genital prolapse is a frecuent condition in clinical practice, defined as the descent of the genital structures and/or elements related with them below the level they usually are. Endopelvic fascia joins organs topelvics walls, and pelvic floor muscles close the pelvis and raise the organs, avoiding the distention of aponeurotic bonds. Are studied 102 patients attenden in the Hospital University of Cartagena with genital prolapse. Ages 32-80 years old. $X^{=}=55.8 \pm 12.3$ years. $74.5 \%$ had a grade III prolapse. Average parity was 7 births, none of them nulipar. The king of prolapse more frequent was cistocele, followed by the total genital prolapse. $30.2 \%$ of the gynecological surgeries made in this Hospital are procedures for the correction of genital prolapse. There weren't any intrasurgery accidents and 30 days complications were $11.8 \%$. Intrahospitalary stay was 3-8 days. The origen of the pelvic floor transtorns is considered as multifactorial, having a great importance the obstetrical traumatism and more recenthy postmenopausic hypoestrogenic states. There's a need of more studies to clarify the influence of nutritional states and familiar tendency in genital prolapse.

KEY WORDS: Genital prolapse, cistocele, histerocele, rectocele, vaginal histerectomy, perineal breakdaun, perineal injury.

\section{Introducción}

El prolapso de los órganos pélvicos es una condición que frecuentemente encuentra el Ginecólogo en su práctica clínica (1). El prolapso genital es definido como el descenso de las estructuras del aparato genital y/o de los elementos relacionados con él, por debajo del nivel que normalmente ocupan (2-3). Se denomina cistocele el descenso de la pared vaginal anterior, la cual se acompaña de descenso de la pared posterior de la vejiga y del trígono. El uretrocele es el descenso de la pared vaginal y de la uretra. El rectocele es el descenso de la pared vaginal posterior acompañada del descenso de la pared rectal anterior. El prolapso de la porción superior de la pared vaginal posterior se denomina enterocele o prolapso del saco de Douglas y suele contener asas intestinales o epiplon. Histerocele es el descenso uterino con o sin elongación del cérvix. El prolapso de la cúpula vaginal consiste en la eversión o descenso de las paredes vaginales después de histerectomía. El prolapso genital total incluye histerocele, cistocele y rectocele (2-6).

\footnotetext{
* Profesor Asistente. Departamento de Ginecología y Obstetricia. Facultad de Medicina. Universidad de Cartagena. Estudiante. Facultad de Medicina. Universidad de Cartagena.
}

La capa superior del piso pélvico es la fascia endopélvica, que une los órganos a las paredes pélvicas, sosteniéndolos. La parte que se une al útero es el parametrio y la que se une a la vagina es el paracolpio. Los parametrios están constituídos por los ligamentos cardinales y uterosacros, que son partes diferentes de una sola masa de tejido. El sostén pélvico incluye vasos sanguíneos, nervios y tejido conectivo fibroso. La capa estructural que sostiene la vejiga es la aponeurosis pubocervical, que está constituída por la pared vaginal anterior y su unión, a través de la aponeurosis endopélvica, a la pared de la pelvis. Existe mayor desarrollo a nivel del cuello vesical y suburetral, siendo de especial importancia para evitar la incontinencia urinaria. La capa inferior son los músculos del piso pélvico, que cierran la pelvis, elevan los órganos y evitan la distensión constante de los ligamentos aponeuróticos ya anotados, estos músculos que proporcionan el sostén son denominados: músculos elevadores del ano, constituidos por dos porciones: la pubococcígea y la ileococcígea, dejando una abertura, denominada hiato urogenital del elevador del ano, por la cual pasan la uretra y la vagina. Los elevadores del ano siempre se están contrayendo. Esta contracción constante sobre la vagina elimina cualquier abertura en el piso 
pélvico a través de la cual pudiera ocurrir prolapso. Existe interacción entre los músculos del piso pélvico y los ligamentos de sostén. Mientras los músculos funcionen de manera normal, el piso pélvico se mantiene cerrado y los ligamentos permanecen sin tensión, estabilizando los órganos por arriba del elevador del ano. Cuando los músculos del piso pélvico se relajan o lesionan, el piso se abre, la vagina es impulsada hacia abajo, las aponeurosis se distienden y con el paso del tiempo el tejido conectivo se elonga y se daña $(1-2,7-8)$.

El parto vaginal y el traumatismo directo o quirúrgico perineal, constituyen factor etiológico importante de prolapso genital, al causar lesiones neuromusculares del piso pélvico (8-9). Los elevadores del ano pueden afectarse por lesión directa del músculo a causa de lesión mecánica o por lesión de su inervación, lo que pudiera llevar a incapacidad para la contracción aunque estén intactas las fibras (7). La debilidad congénita de los medios de fijación del aparto genital es causa de un pequeño porcentaje de casos de prolapso genital (10-11).

En la génesis del prolapso intervienen una serie de factores predisponentes tales como: edad avanzada, el hipoestrogenismo subsecuente a la post-menopausia, factores genéticos, raciales, nutricionales, laborales, estilos de vida, la obesidad, las variaciones en la composición del colágeno, la acentuación del cul de sac y la existencia de enfermedades como el EPOC y el estreñimiento crónico, en las cuales el aumento de la presión intraabdominal en forma continua, lleva a deterioro del sistema músculoaponeurótico pélvico $(1,3-4,9,11)$.

Estudios histológicos de la musculatura del piso pélvico en pacientes con prolapso genital revelan evidencias de denervación extensa, asociada a atrofia de las fibras musculares. Múltiples estudios han documentado alteraciones en mediciones electrofisiológicas del piso pélvico en pacientes con severos prolapsos de los órganos pélvicos e incontinencia de esfuerzo (1).

Nos proponemos determinar algunas características epidemiológicas (incidencia con respecto a la totalidad de los procedimientos quirúrgicos realizados, el área de residencia, la distribución por grupos etáreos, la paridad, la sintomatología, tipos y grados de prolapso, el manejo, la estancia hospitalaria y la morbi-mortalidad), del prolapso genital en el Hospital Universitario de Cartagena.

\section{Material y método}

Se realizó un estudio descriptivo, retrospectivo, en el cual se revisaron todas las historias clínicas de las pacientes que fueron hospitalizadas con diagnóstico de prolapso genital, en el servicio de Ginecología del Hospital Universitario de Cartagena, Colombia, durante el período comprendido entre junio 1o. de 1993 y el 31 de mayo de 1994.

Se tomaron los datos referentes a: edad, residencia de la paciente, paridad, sintomatología, tipo y grado de prolapso, tratamiento realizado, estancia en el hospital y morbimortalidad intra y post-operatoria, hasta los 30 días después de la cirugía. Los datos fueron consignados en una tabla maestra, almacenados y tabulados en un PC con ayuda del programa EPI INFO 5.

\section{Resultados}

En el período escogido para revisar, fueron ingresadas al servicio de Ginecología del Hospital Universitario de Cartagena, Colombia, 102 pacientes con diagnóstico de prolapso genital.

La edad de las pacientes estaba comprendida entre los 32 y 80 años de edad con $\mathrm{X}^{=}=55.8$ y $\mathrm{SD}=12.3$ años. $\mathrm{La}$ mayoría de las pacientes (30.4\%) estaban en el rango etáreo 51-60 años. El 68\% de las pacientes eran mayores de 51 años de edad y sólo el $13 \%$ eran menores de 40 años de edad. (Figura 1).

66 pacientes $(64.7 \%)$ residían en áreas rurales de los Departamentos de Bolívar, Córdoba o Sucre y 36 (35.3\%) de barrios de estratos sociales bajos de la ciudad de Cartagena.

76 pacientes $(74.5 \%)$ tenían prolapso Grado III, 24 pacientes $(23.6 \%)$ tenían prolapso Grado II y 2 pacientes $(1.9 \%)$ tenían grado I, asociado a incontinencia urinaria de esfuerzo.

El rango de paridad se encontró entre 1 y 14 partos y el promedio fue de 7 partos. 76 pacientes $(74.5 \%)$ tenían como antecedente más de seis partos. En cada uno de los grupos de número de partos se encontró predominio del prolapso Grado III. Ninguna de las pacientes ingresadas era nulípara (Figura 2).

\section{Figura 1 \\ PROLAPSO GENITAL EN EL HOSPITAL UNIVERSITARIO DE CARTAGENA}

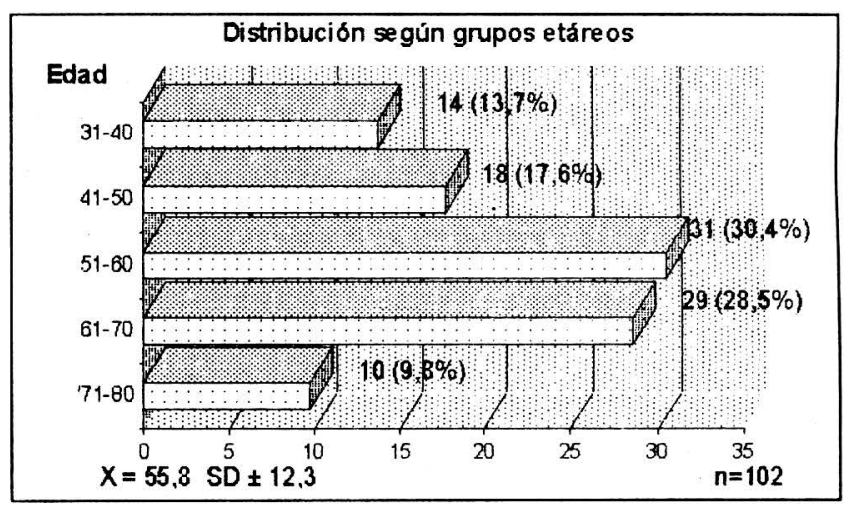

Figura 2

PROLAPSO GENITAL EN EL HOSPITAL UNIVERSITARIO DE CARTAGENA

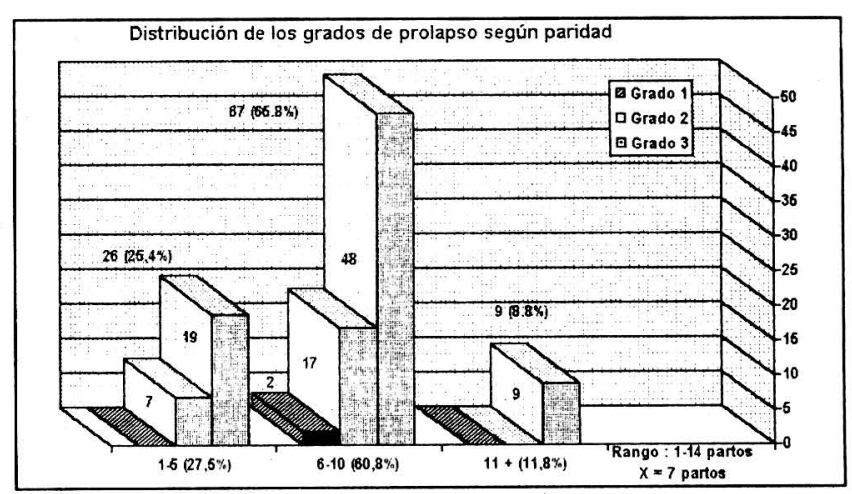


La sintomatología más frecuentemente relatada fue la sensación de cuerpo extraño vaginal $(68.6 \%)$, seguido de la incontinencia urinaria de esfuerzo $(11.8 \%)$. (Tabla 1$)$. Al desglosar la sintomatología según grado de prolapso, se observa que un mayor número de síntomas estaban presentes a medida que el prolapso genital era de mayor grado. Igual con respecto a la sintomatología y tipos de prolapso, entre más tipos de prolapso coexistan es mayor la sintomatología.

Los tipos de prolapso más frecuentes fueron: Cistorectocele 49 casos (48\%), seguido del prolapso genital total en 46 pacientes (45\%) y menos frecuente el uretrocele y el enterocele. (Tabla 2).

El tratamiento fue quirúrgico en todos los casos, realizándose técnicas de acuerdo al tipo de prolapso. La tabla 3, presenta las técnicas quirúrgicas realizadas para los diferentes tipos de prolapso genital. 338 cirugías ginecológicas fueron adelantadas en el período revisado, de las cuales $102(30.2 \%)$ correspondieron a procedimientos para corrección del prolapso genital.

No se presentaron accidentes intraoperatorios. Sólo se presentó una complicación post-quirúrgica inmediata que consistió en una monoparesia derecha e hipoestesia transitoria por posible anafilaxia o reacción medular local, al anestésico utilizado. No se presentaron complicaciones inmediatas inherentes a la técnica que ameritaran reintervención. Complicaciones post operatorias tardías, evaluadas las pacientes a los 30 días post-quirúrgicos, se encontraron en 12 pacientes (11.8\%). (Tabla 4). La estancia hospitalaria post-operatoria fue de 3 a 8 días, con un promedio de 4 días. No se presentó mortalidad intraoperatoria ni post-operatoria.

Tabla 1

\section{SINTOMAS SOBRESALIENTES}

\begin{tabular}{|ccc|}
\hline Síntomas & No. & $\%$ \\
\hline Sensación de cuerpo Extraño & 70 & 68.6 \\
I.U.E.* Exclusivamente & 12 & 11.8 \\
I.U.E.* + Sens. cuerpo extraño & 9 & 8.8 \\
Dolor & 7 & 6.9 \\
Sangrado & 4 & 3.9 \\
\hline Total & 102 & 100 \\
\hline
\end{tabular}

*I.U.E. = Incontinencia urinaria de esfuerzo.

Tabla 2

\section{DISTRIBUCION SEGUN TIPOS DE PROLAPSO} GENITAL

\begin{tabular}{|lcc|}
\hline Tipos de prolapso & No. & $\%$ \\
\hline Cistocele + Rectocele & 49 & 48.0 \\
Prolapso Genital Total & 46 & 45.0 \\
Histerocele & 3 & 3.0 \\
Prolapso de Cúpula & 2 & 2.0 \\
Uretrocele & 1 & 1.0 \\
Enterocele & 1 & 1.0 \\
\hline Total & 102 & 100 \\
\hline
\end{tabular}

Tabla 3

\section{TIPO DE PROLAPSO GENITAL Y TECNICA QUIRURGICA REALIZADA}

\begin{tabular}{|c|c|c|c|}
\hline Tipo prolapso & Técnica quirúrgica & No. & $\%$ \\
\hline Prolapso Total & $\begin{array}{c}\text { Histerectomía } \\
\text { vaginal + Colporrafía }\end{array}$ & 44 & 43.0 \\
\hline Prolapso total & Colpocleisis de Leffort & 2 & 2.0 \\
\hline Cistocele + Rectocele & $\begin{array}{c}\text { Colporrafía } \\
\text { anterior y posterior }\end{array}$ & 49 & 48.0 \\
\hline Uretrocele & Cirugía de Tanago & 1 & 1.0 \\
\hline Histerocele & $\begin{array}{c}\text { Histerectomía } \\
\text { vaginal + Colporrafía }\end{array}$ & 1 & 1.0 \\
\hline Histerocele & Cirugía de Manchester & 2 & 2.0 \\
\hline Enterocele & Cierre del enterocele & 1 & 1.0 \\
\hline Prolapso de cúpula & $\begin{array}{l}\text { Suspensión de } \\
\text { cúpula vía vaginal }\end{array}$ & 2 & 2.0 \\
\hline Total & & 102 & 100 \\
\hline
\end{tabular}

Tabla 4

\section{COMPLICACIONES A LOS 30 DIAS POST-OPERATORIOS}

\begin{tabular}{|lcr|}
\hline Complicaciones & No. & $\%$ \\
\hline Infección de cúpula vaginal & 8 & 7.8 \\
Dehiscencia de sutura & 1 & 1.0 \\
Granuloma de sutura vaginal & 1 & 1.0 \\
Incontinencia urinaria al esfuerzo & 2 & 2.0 \\
Sin complicaciones & 90 & 88.2 \\
\hline Total & 102 & 100 \\
\hline
\end{tabular}

\section{Comentarios}

La mayoría de las pacientes que presentaron prolapso genital eran mayores de 50 años de edad, lo cual se correlaciona con resultados obtenidos por otros investigadores en Colombia (3) y en otros países (9, 12-13). Es reconocido que la relajación del piso pélvico es un fenómeno progresivo, que en la post-menopausia por la condición hipoestrogénica presente, se acelera, facilitando el establecimiento de la sintomatología. Recientes investigaciones $(11,14-15)$ han demostrado los beneficios de la terapia estrogénica sustitutiva en pacientes añosas, pero aún se desconoce si es un efecto directo de los estrógenos. Sin embargo los transtornos del piso pélvico no son exclusivos de las mujeres de edad avanzada $(4,10-11)$. En el estudio encontramos que las pacien- 
tes tenían edad promedio de 55 años, mientras que en un estudio realizado en Dakar se reporta edad promedio 30 años, y los autores resaltan el papel que puede jugar el trauma obstétrico en la génesis del prolapso genital en Africa (16).

La mayoría de las pacientes estudiadas residían en áreas rurales, grupo poblacional que suele tener una deficiente calidad de atención obstétrica, ya que es corriente tener los partos con personal empírico, y además es común la elevada paridad. Este último evento está demostrado en la presente evaluación, donde la paridad promedio de las pacientes fue de 7.

El prolapso genital se clasifica en: Grado I, cuando las estructuras descienden sin llegar al introito vaginal. Grado II, cuando descienden hasta el introito vaginal. Grado III, si salen más allá del introito vaginal (2-4). Los grados avanzados de prolapso encontrados al momento del ingreso, son debidos generalmente a una consulta tardía. Existe relación directa entre prolapso genital grado III y el bajo nivel socio-económico-cultural de las pacientes. Llegar a estas condiciones avanzadas de prolapso genital pueden ser reflejo de un deficiente nivel de atención obstétrica como parte de una muy baja oportunidad de atención en salud, de una deficiente educación en salud reproductiva, que conlleva un pobre conocimiento y escaso uso de las tecnologías modernas contraceptivas, para una subsecuente elevada paridad. Hoy día se entiende la influencia que ejerce el parto vaginal, técnicamente mal atendido, en la producción del daño pélvico, al ocurrir lesión neuromuscular $(1,9,12-13,16)$. Existe relación entre multiparidad y prolapso genital, no obstante existen en la literatura casos de prolapso genital en mujeres vírgenes y nulíparas jóvenes, así como en primigestas (17-18). La atención obstétrica será de calidad óptima, si se realiza la protección perineal adecuada, se evitan las distensiones y/o desgarros y se practica episiotomia y episiorrafía con una técnica depurada.

El origen de los trastornos del piso pélvico se puede considerar multifactorial. No obstante a que el daño neuromuscular influye en la evolución de la disfunción del piso pélvico, algunas investigaciones demuestran que anomalías del tejido conectivo, podrían explicar el prolapso genital en nulíparas o su recidiva en las que han sido sometidas a cirugías correctoras (19).

La sintomatología referida por las pacientes corresponde a lo anotado en la literatura y se correlaciona con el tipo y grado de prolapso. Los síntomas por los cuales consultan las pacientes con prolapso genital son frecuentemente: dolor o sensación de peso en hipogastrio o región lumbar, sensación de cuerpo extraño intravaginal, transtornos urinarios, constipación o tenesmo, dispareunia, leucorrea, disconfor vaginal y en los casos avanzados, salida de mas por genitales externos o sangrado, éste debido a laceraciones en la mucosa vaginal prolapsada (2-5).

El diagnóstico se basa principalmente en la inspección, ordenándole a la paciente pujar para comprobar la presencia del prolapso (3-4). Aproximadamente del 10 al $15 \%$ de las mujeres con prolapso inicial de los órganos pélvicos desarrollan sintomatología que requiere diagnóstico y cirugía (1).

Diversos soportes y pesarios fueron utilizados mucho antes de la era quirúrgica moderna. Los pesarios causaron alivio de la sintomatología en muchas mujeres y tuvieron su mayor popularidad a mediados del siglo XIX. Para la segunda mitad de este siglo se realizaron los primeros intentos quirúrgicos para tratar el prolapso genital (2). El tratamiento de elección es el quirúrgico, existiendo porcentajes importantes de falla en el acto operatorio o de recidivas. Más de un centenar de procedimientos quirúrgicos han sido descritos para el manejo de la incontinencia urinaria (1). En nuestro medio la Colporrafía anterior suele utilizarse para el cistocele y uretrocele. Colporrafía posterior para la corrección del rectocele y los desgarros del periné, Culdoplastia de McCall para obliteración del fondo de saco de Douglas mediante la aproximación de los ligamentos uterosacros. Operación de Manchester o amputación del cuello para tratamiento de la elongación del cérvix. Histerectomía vaginal con colporrafía anterior y posterior para el prolapso total. Colpocleisis de Lefort para cierre total de vagina, en mujeres de edad avanzada y $\sin$ coitos (2-5). No existe procedimiento quirúrgico perfecto para el tratamiento del prolapso genital. Las técnicas y vías deberán elegirse de acuerdo con las necesidades específicas de cada paciente, teniendo en cuenta los siguientes objetivos: aliviar los síntomas, restituir la anatomía y restablecer la funcionabilidad genital (20).

La estancia hospitalaria post-cirugía correctora de prolapso fue relativamente corta, a pesar de la edad avanzada que tenían las pacientes. La complicación infecciosa local fue la más frecuente y no se presentó mortalidad en la población evaluada. Aunque se ha escrito mucho acerca del origen de los trastornos del piso pélvico, incluyendo recientes valoraciones sobre la calidad de la colagena de los tejidos de sostén de los órganos, se carece aún de datos científicos para valorar cuáles teorías son correctas que expliquen su presencia o su recidiva (2). No se tiene información básica acerca de la prevalencia y evolución natural del prolapso genital en la población (21). Información acerca de las diferencias raciales, la influencia de los estados nutricionales y tendencias familiares es escasa.

\section{BIBLIOGRAFIA}

1. Theofrastous JP. Pelvic organ prolapse: relationship to pelvic floor dysfunction. Postgraduate Obstetrics and Gynecology. 1994; 14(15): $1-8$.

2. Thompson JD. Anomalias en la posición del útero. En: Thompson JD, Rock JA. Ginecología quirúrgica. Te Linde. 7a. Edición. Editorial Médica Panamericana. Buenos Aires, 1993; 746-776.

3. Luna J.Epidemiología del prolapso genital. Rev. Col. Obstet. Gynecol. 1985; 36: 307-322.
4. González J. Prolapso genital. Ginecología. 6a. Edición. MassonSalvat. Barcelona. 1993; 255-263.

5. Jones h. Wentz A. Burnett L. Tratado de Ginecología de Novak. Interamericana Mc Graw-Hill. México 11a. Edición. 1994; 403-408.

6. Delancey JOL. Anatomic aspects of vaginal eversion after hysterectomy. Am. J. Obstet. Gynecol. 1992; 166: 1717-1722.

7. Delancey JOL. Anatomía y biomecánica del prolapso genital. Clínica Obstétrica y Ginecológica. 1993; 4: 845-855. 
8. Allen RE., Hosker GL., Smith ARB., Warrell DW. Pelvic floor damage and childbirth: a neurophysiologic study. Br. J. Obstet. Gynaecol. 1990; 97: 770-778.

9. Younis N., Khattab H., Zurayk $\mathrm{H}$ et al. A community study gynecological and related morbidities in rural Egypt. Stud Fram Plann 1993; 24: 175-186.

10. Bader D., Davidovitch M., Berger A. Genital prolapse in a preterm femele infant J. Perinatol. 1993; 13: 159-161.

11. Bayatpour M., McCann J., Harris T., Phelps H. Prolapso genital neonatal. Pediatrics 1992; 34: 157-158.

12. Santos M., Cunha N., Marques-Neto A et al. Prolapso uterino: Estudio retrospectivo de 423 casos. J. Bras. Ginecol. 1987; 97: 305-308.

13. Sfar E., Marai K., Chelli H., Kharouf M et al. Resultats au centre de metermité et neónatologie de Rabta Tunis du traitmment. Rev. Fr. Gynecol. Obstet. 1992; 87: 7-11.

14. Brincat M., Versi E., Moniz C., Magos A et al. J skin collagen changes in postmenopausal women receiving different regimens of estrogen. Obstet. Gynecol. 1987; 70: 123-127.
15. Castelo-Branco C., Durán M., González-Merlo J. Skin collagen changes to age and hormone replacement therapy. Maturitas. 1992; 15: 113-119.

16. Dia A., Tource C., Diop M et al. Prolapse genital a Dakar. Dakar-Med 1991; 36: 39-46.

17. Smith A., Hosker G., Warrell D. The role of parcial denervation of the pelvic floor in the etiology of genitourinary prolapse and stress incontinence of urine: a neurophysiological study. Br. J. Obstet. Gynecol. 1989; 96: 24-28.

18. Sepúlveda W., Cabrera J. Prolapso uterino en primigesta. Rev. Chil. Obstet. Gynecol. 1984; 49: 111-114.

19. Norton P., Boyd C., Deak S. Abnormal collagen ratios in women with genito-urinary prolapse. Neuro-Urodyn. 1992; 11: 2-4.

20. Nichols D. Surgery for pelvic floor disorders. Surg. Clin. North. Am. 1991; 71: 927-946.

21. Harris TA., Bent-AE. Genital prolapse with and without urinary incontinence. J. Reprod. Med. 1990; 35(8): 792-798. 